\title{
Suppression of STN1 enhances the cytotoxicity of chemotherapeutic agents in cancer cells by elevating DNA damage
}

\author{
QING ZHOU ${ }^{1}$ and WEIHANG CHAI ${ }^{1,2}$ \\ ${ }^{1}$ Department of Biomedical Sciences, Elson S. Floyd College of Medical Sciences; \\ ${ }^{2}$ School of Molecular Biosciences, Washington State University, Spokane, WA 99210, USA
}

Received May 10,2015; Accepted April 22, 2016

DOI: $10.3892 / \mathrm{ol} .2016 .4676$

\begin{abstract}
DNA damage-inducing agents are among the most effective treatment regimens in clinical chemotherapy. However, drug resistance and severe side effects caused by these agents greatly limit their efficacy. Sensitizing malignant cells to chemotherapeutic agents has long been a goal of chemotherapy. In the present study, suppression of STN1, a gene important for safeguarding genome stability, potentiated the anticancer effect of chemotherapeutic agents in tumor cells. Using multiple cancer cells from a variety of origins, it was observed that downregulation of STN1 resulted in a significant decrease in the half maximal inhibitory concentration values of several conventional anticancer agents. When cells are treated with anticancer agents, STN1 suppression leads to a decline in colony formation and diminished anchorage-independent growth. Furthermore, it was additionally observed that STN1 knockdown augmented the levels of DNA damage caused by damage-inducing agents. The present study concluded that suppression of STN1 enhances the cytotoxicity of damage-inducing chemotherapeutic agents by increasing DNA damage in cancer cells.
\end{abstract}

\section{Introduction}

Besides surgery and radiotherapy, chemotherapy is regarded as the most effective means of current clinical treatment for cancer. A large group of chemotherapeutic drugs target rapidly-dividing cancer cells by directly damaging genomic

Correspondence to: Professor Weihang Chai, Department of Biomedical Sciences, Elson S. Floyd College of Medical Sciences, Washington State University, 412 E Spokane Falls Boulevard, Spokane, WA 99210, USA

E-mail:wchai@wsu.edu

Abbreviations: CPT, camptothecin; DMSO, dimethylsulfoxide; OBFC1, oligonucleotide/oligosaccharide binding fold containing protein 1;MTT,3-(4,5-dimethylthiazol-2-yl)-2,5-diphenyltetrazolium bromide

Key words: STN1, chemosensitization, genome stability, DNA damage
DNA, thereby inhibiting tumor growth $(1,2)$. Cancer cells have high proliferation rates and replicate their DNA rapidly, making them highly susceptible to DNA damage, as replicating damaged DNA increases the likelihood of cell death $(1,3)$. Commonly used drugs in this group include: Cisplatin, camptothecin, etoposide, bleomycin, doxorubicin and gemcitabine (1).

Despite the success of chemotherapy, drug resistance is a major obstacle to successful chemotherapy $(4,5)$. Resistance to chemotherapy results in increased tumor growth and decreased patient survival. In addition, severe side effects resulting from adverse toxicity to non-target tissues are often observed in patients $(4,6)$. These drawbacks pose a significant impediment to the development of desired chemotherapy regimens. A promising method of reducing severe side effects may be to develop approaches that sensitize cancer cells to current chemotherapies.

For chemotherapy that is based on inducing DNA damage, modulating DNA damage response or DNA repair is highly desirable for enhancement of the sensitivity of cancer cells to these therapies $(4,7)$. This may be achieved by specifically targeting factors involved in damage response and DNA repair pathways. Previous studies have demonstrated that the CTS telomere maintenance complex component 1 (CTC1)-STN1-TEN1 (CST) complex may have a role in maintaining genome stability (8-10). CTC1 and STN1 were originally identified as accessory factors of DNA polymerase alpha (Pol $\alpha)$ and were named as AAF132 and AAF44, respectively (11). STN1 is also known as oligonucleotide/oligosaccharide-binding fold containing 1 (OBFC1) (12). Deficiencies in components of the CST complex induces DNA damage (8-10), suggesting that CST may have an important role in safeguarding genome stability. It appears that such a role is particularly prominent in the presence of replication stress $(9,10)$. After cells are treated with hydroxyurea, which depletes the nucleotide pool and induces replication stress, deficiency in CST leads to delayed recovery of stalled replication $(9,10)$. It has been postulated that one significant function of the CST complex may be to promote efficient replication of difficult-to-replicate sequences throughout the genome, perhaps by facilitating efficient restart of stalled replication (8-10).

Several previous studies have demonstrated that CST also has a significant role in telomere protection (8-10,13-17). Telomeres are highly complex nucleoprotein structures located at the ends of linear eukaryotic chromosomes, which are 
conserved in organisms ranging from unicellular eukaryotes to mammals $(18,19)$. Human telomeres consist of tandem repeats of the sequence TTAGGG, which typically extend up to $10-15 \mathrm{~kb}$. Potential functions of telomeres include prevention of chromosome degradation, end-to-end fusions, rearrangements and chromosome loss $(18,20,21)$. In normal somatic cells, progressive telomere shortening occurs during each cell division $(18,19)$. When telomere length becomes critically short, cells senesce and growth arrests. Cancer cells require mechanisms to maintain their telomeres in order to continue dividing indefinitely $(18,22)$. Disruption of telomere maintenance in cancer cells prevents cells from uncontrolled proliferation (23). Approximately $85-90 \%$ of cancer cells activate the telomerase gene, which utilizes its reverse transcriptase activity to add telomere repeats at chromosome ends to counteract telomere shortening $(18,19,24)$. Telomerase inhibition or inactivation in telomerase-expressing cancer cells drives telomere shortening, inducing growth arrest or death of tumor cells (18).

The CST complex binds to telomeres and is important for several aspects of telomere maintenance. It promotes efficient replication of telomeric DNA. RNA interference (RNAi)-mediated suppression of STN1 and CTC1 elevates defects in telomere replication, leading to increased telomere loss (8-10). CST interacts with Pol $\alpha$ and is required for replenishing the telomere $\mathrm{C}$ strand following replication. Loss of CST leads to elongated G-overhangs $(9,16,25)$. In certain cancer cell lines, CST binds to single-stranded G-rich overhangs at telomere ends and prevents telomerase from excessively extending telomere DNA (15).

The multiple significant functions of CST in maintaining genomic stability prompted the present study to postulate that suppression of CST may act synergistically with chemotherapeutic agents that induce genome instability, in particular to those agents that cause DNA damage. Using various cell proliferation assays, the present study observed that depletion of STN1 enhanced the cytotoxicity of bleomycin, camptothecin and etoposide in various cancer cells from diverse origins including the lung, breast and cervix. Comet assay additionally revealed that STN1 suppression significantly elevated DNA damage levels in cells treated with damaging agents, suggesting that CST may have an important role in repairing DNA damage.

\section{Materials and methods}

Materials. The following chemotherapeutic agents were used: Camptothecin (Sigma-Aldrich, St. Louis, MO, USA), etoposide (Sigma-Aldrich), bleomycin (Sigma-Aldrich) and meso-tetra (N-methyl-4-pyridyl) porphine tetra tosylate (TMPyP4; EMD Millipore, Darmstadt, Germany). All agents were dissolved in dimethyl sulfoxide (DMSO) and stored under sterile conditions at $-20^{\circ} \mathrm{C}$ in the dark. The vehicle (DMSO) was utilized as a control with a final concentration of $<0.1 \%$, which had no influence on cell growth.

Cell culture. Cells obtained from American Type Culture Collection (Manassas, VA, USA) were passaged in Dulbecco's modified Eagle's medium (DMEM; GE Healthcare Life Sciences, Logan, UT, USA) supplemented with $10 \%$ cosmic calf serum (GE Healthcare Life Sciences) at $37^{\circ} \mathrm{C}$ in a humidified atmosphere containing $5 \% \mathrm{CO}_{2}$ for $<6$ months. No antibiotics were added to the medium to avoid stress.

Antibodies. The following primary antibodies were utilized: Polyclonal rabbit anti-OBFC1 (1:500 dilution; cat no. sc-135364; Santa Cruz Biotechnology, Inc., Dallas, TX, USA) and monoclonal mouse anti- $\beta$-actin (1:60,000 dilution; cat no. A2228; Sigma-Aldrich). The secondary antibody was horseradish peroxidase-conjugated polyclonal goat anti-mouse immunoglobulin (Ig)G (cat no. 554002; BD Biosciences, San Jose, CA, USA) or anti-rabbit IgG (cat no. PI-1000; Vector Laboratories, Inc., Burlingame, CA, USA).

RNAi. STN1 small hairpin (sh)RNA sequences targeting GCUUAACCUCACAACUUAA (shStn1-2) (9) and GGA CUGCCAGAAACCAAAT (shStn1-4) were cloned into pSIREN-retro-puro (Clontech Laboratories, Inc., Mountainview, CA, USA). Control shRNA targeted luciferase and the sequence was CGUACGCGGAAUACUUCGA (shLuc) (Thermo Fisher Scientific, Inc., Waltham, MA, USA). Infection and selection were performed as previously described (9).

3-(4,5-dimethylthiazol-2-yl)-2,5-diphenyltetrazolium bromide (MTT) assay. MTT assay was employed to evaluate cell viability. Briefly, cells were seeded into 96 -well multiplates at a density of $1 \times 10^{4} / \mathrm{ml}$. Following overnight incubation, cells in triplicate wells were treated with camptothecin, etoposide or bleomycin at the indicated concentrations $(5,50$ and $500 \mathrm{nM})$ for 5 days, and subsequently incubated with $100 \mu \mathrm{l}$ of $0.5 \mu \mathrm{g} / \mathrm{ml}$ MTT for an additional $4 \mathrm{~h}$ at $37^{\circ} \mathrm{C}$. MTT was subsequently removed, and DMSO was added to dissolve the resulting formazan crystals. The light absorption was measured at $570 \mathrm{~nm}$ with a microplate spectrophotometer (BioTek Instruments, Inc., Winooski, VT, USA). Effects of chemicals on cell survival were assessed by half maximal inhibitory concentration $\left(\mathrm{IC}_{50}\right)$ values (the concentration resulting in $50 \%$ inhibition of cell growth).

Colony formation assay. H1299 shLuc, shSTN1-2 and shSTN1-4 cells, and HeLa shLuc, shSTN1-2 and shSTN1-4 cells (2 days after puromycin selection) were seeded into 6 -well plates at a density of 100 cells/well and incubated overnight. On the following day, cells were treated with various concentrations $(500,50$ and $5 \mathrm{nM})$ of each testing drug (camptothecin, etoposide and bleomycin) at $37^{\circ} \mathrm{C}$. Identical treatments were repeated every 4 days. After 10 days of incubation, the medium was removed and cell colonies were fixed and stained with crystal violet solution $(0.1 \%$ crystal violet, $1 \%$ methanol and $1 \%$ formaldehyde). Colonies with $>50$ cells were counted and the percentage of drug-treated colonies relative to DMSO-treated control colonies was calculated.

Soft agar colony formation assay. Soft agar assays were performed in 6-well plates. Equal volumes of 1.2\% agar were mixed with 2X DMEM and $1 \mathrm{ml}$ of this mixture was added into each well as the base layer. Plates were chilled at $4^{\circ} \mathrm{C}$ until the agar solidified. Subsequently, $1 \times 10^{4}$ cells that were suspended in 1X DMEM containing a gradient concentration $(5,50,500 \mathrm{nM})$ of drugs, and then mixed with equal volume of 
Table I. $\mathrm{IC}_{50}$ values of camptothecin, etoposide, bleomycin and TMPyP4 in H1299 cells with either STN1 knockdown or control.

$$
\mathrm{IC}_{50} \text { value, } \mu \mathrm{M}
$$

\begin{tabular}{lcccr}
\cline { 2 - 4 } Cell type & Camptothecin & Etoposide & Bleomycin & TMPyP4 \\
\hline shLuc & $0.57 \pm 0.01$ & $0.43 \pm 0.29$ & $7.10 \pm 1.60$ & $8.65 \pm 0.44$ \\
shStn1-2 & $0.20 \pm 0.03$ & $0.13 \pm 0.10$ & $2.36 \pm 0.94$ & $5.76 \pm 1.76$ \\
shStn1-4 & $0.18 \pm 0.17$ & $0.11 \pm 0.06$ & $3.07 \pm 0.92$ & $5.14 \pm 1.39$
\end{tabular}

$\mathrm{IC}_{50}$, half maximal inhibitory concentration; TMPyP4, meso-Tetra ( $\mathrm{N}$-methyl-4-pyridyl) porphine tetra tosylate; sh, small hairpin; shLuc, shRNA targeting luciferase.

$0.7 \%$ agar. A total of $1 \mathrm{ml}$ of the mixture was poured as the top layer. Once the growth layer congealed, cells were permitted to grow at $37^{\circ} \mathrm{C}$ for 10 days and total colonies were counted and normalized against untreated cells.

Comet assay. DNA damage levels in wild-type and STN1 knockdown cells following exposure to drugs were assessed using the comet assay. $\mathrm{H} 1299$ cells were treated with $1 \mu \mathrm{M}$ of each testing agent for $6 \mathrm{~h}$, while HeLa cells were treated with $3 \mu \mathrm{M}$ of each agent for $8 \mathrm{~h}$. Immediately subsequent to treatment, $10 \mu \mathrm{l}$ cell suspension was mixed with $50 \mu 10.5 \%$ low-melting agarose, spread onto a Cometslide (Trevigen, Gaithersburg, MD, USA), and pre-coated with $100 \mu \mathrm{l}$ of $1 \%$ normal-melting agarose. Cells were lysed by immersing the slides in a freshly prepared lysis solution [2.5 M NaCl, $100 \mathrm{mM}$ ethylenediaminetetraacetic acid (EDTA), 10 mM Tris, $1 \%$ Triton X-100, 10\% DMSO; pH 10] at $4^{\circ} \mathrm{C}$ for $2 \mathrm{~h}$. Following lysis, slides were washed 3 times in $1 \mathrm{X}$ phosphate-buffered saline and placed in a gel electrophoresis apparatus with freshly prepared electrophoresis buffer $(1 \mathrm{mM}$ EDTA, $300 \mathrm{mM} \mathrm{NaOH}$; pH 13) for $25 \mathrm{~min}$ to allow DNA unwinding. Electrophoresis was subsequently performed at $20 \mathrm{~V}$ with a starting current of $300 \mathrm{~mA}$ for $20 \mathrm{~min}$. Subsequently, slides were neutralized with $0.4 \mathrm{M}$ Tris ( $\mathrm{pH} 7.5) 3$ times, stained with 1X SYBR Gold solution (Thermo Fisher Scientific, Inc.), and viewed under an epifluorescence microscope (Axio Imager M2; Zeiss AG, Oberkochen, Germany). Data was analyzed and presented in terms of comet occurrence (\% cells containing comets) and tail extent moment (tail length $\mathrm{x}$ tail intensity). Comets were scored visually without using analysis software, classifying them as belonging to one of five classes according to the tail intensity. Each comet class was given a value between 0 and 4: A score of 0 indicated undamaged cells (i.e., no comet) while a score of 4 indicated maximum damage. The parameter 'comet occurrence' was calculated from this classification and measured in arbitrary units. The comet occurrence was calculated using the following equation: [(\% of cells in class $0) \times 0+(\%$ of cells in class $1 \times 1+(\%$ of cells in class 2$) \times 2+(\%$ of cells in class 3$) \times 3+(\%$ of cells in class 4$) \times 4]$ / total cell number (26). Tail length and tail intensity were evaluated by OpenComet version 1.3 software (www.cometbio.org). A total of 100 cells per sample were selected from random fields and counted for comets. A single investigator analyzed all slides to minimize scoring variation.

Statistical analysis. Two-tailed Student's t-tests were performed in Microsoft Excel 97-2003 (Microsoft Inc., Redmond, WA,
USA) to calculate $\mathrm{P}$-values. $\mathrm{P}<0.05$ was considered to indicate a statistically significant difference.

\section{Results}

STN1 suppression enhances the cytotoxicity of commonly used chemotherapeutic reagents in various cancer cells. The CST complex has a significant role in telomere length control as well as in promoting efficient replication of difficult-to-replicate genomic sequences $(8-10,15)$. Previously the present authors and others have demonstrated that suppression of CST in human cells results in increased staining of the phosphorylated form of histone $\mathrm{H} 2 \mathrm{~A}$, member $\mathrm{X}$ in telomeric and non-telomeric regions in the absence of exogenous damage $(8,9)$, suggesting that CST deficiency may lead to genome instability. However, the DNA damage level induced by CST deficiency is insufficient to elicit marked growth defects in common cancer cell lines $(9,10)$, suggesting that cancer cells may be able to tolerate low levels of DNA damage induced by CST deficiency. We hypothesize that, in the presence of exogenous DNA damaging agents, CST deficiency may augment DNA damage levels, therefore sensitizing cancer cells to damage-inducing agents. To test this, the present study investigated the differences in the sensitivity between wild-type and STN1-deficient cells using the MTT assay. The present study focused on STN1, as a previous study indicated that STN1 is required for formation of the CST complex (27). In agreement with this, the present study also observed that $\mathrm{CTC1}$ expression was reduced when STN1 was absent (data not shown). Using shRNA, the present study initially established cell lines with stable STN1 depletion (Fig. 1). Cancer cell lines from various origins, including the H1299 human non-small lung carcinoma cell line, HeLa human cervical epithelial adenocarcinoma cell line and human breast adenocarcinoma cell line MDA-MB231, were infected with retroviruses expressing two distinct STN1 shRNAs (shStn1-2 and shStn1-4). Following selection, puromycin-resistant cells were collected and immediately treated with chemotherapeutic agents that are commonly used in clinical treatment. STN1 depletion was confirmed by western blotting (Fig. 1). A total of 4 chemotherapeutic agents were used: Camptothecin (CPT), etoposide, bleomycin and TMPyP4. Among them, CPT, bleomycin and etoposide are potent DNA damage inducers (28-33). TMPyP4 is a cationic porphyrin compound that binds to and stabilizes a particular DNA secondary structure known as G-quadruplexes, which are formed by guanine residues through Hoogsteen hydrogen 
A

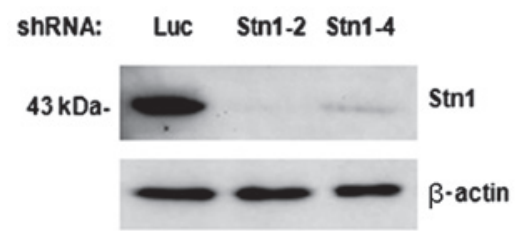

B

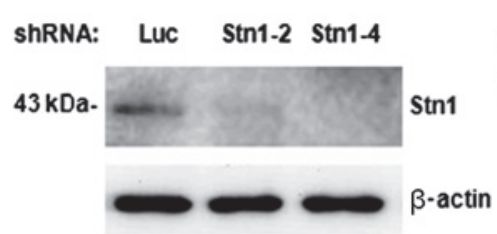

C ShRNA: LuC Stn1-2 Stn1-4

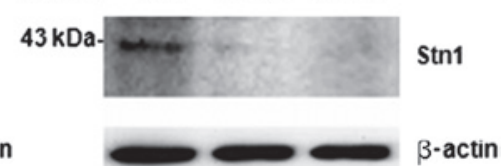

Figure 1. Western blot of STN1 knockdown showing STN1 expression in (A) H1299, (B) HeLa and (C) MDA-MB231 cells. Cells were infected with a retrovirus expressing shRNAs and selected for puromycin resistance. After selection was completed, cells were pooled and subjected to western blotting. shRNA, small hairpin RNA; shLuc, shRNA targeting luciferase.
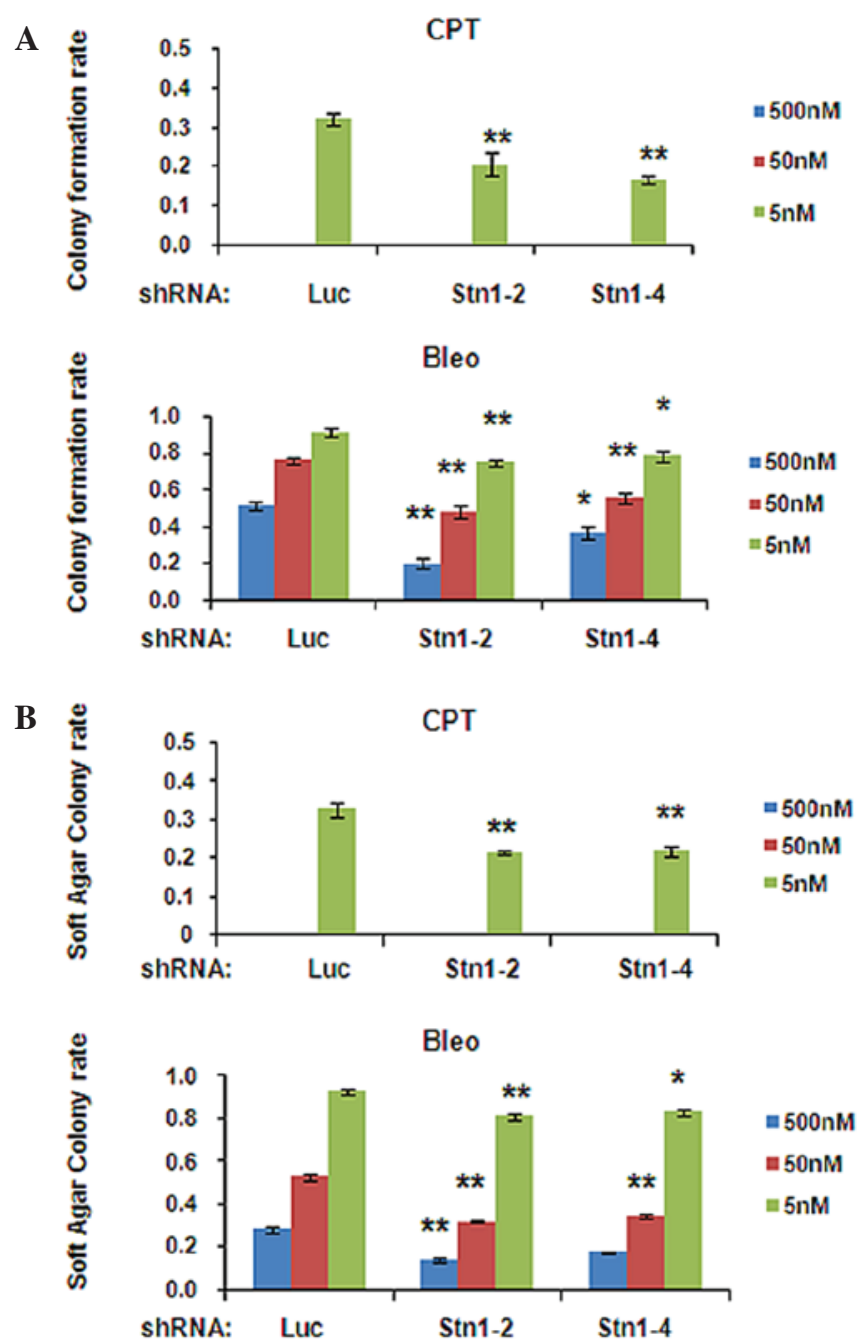

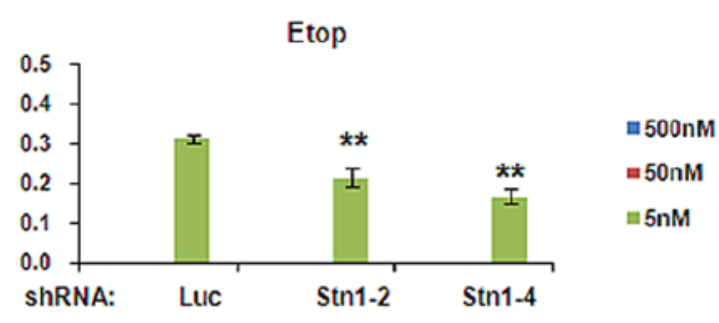

TMPyP4
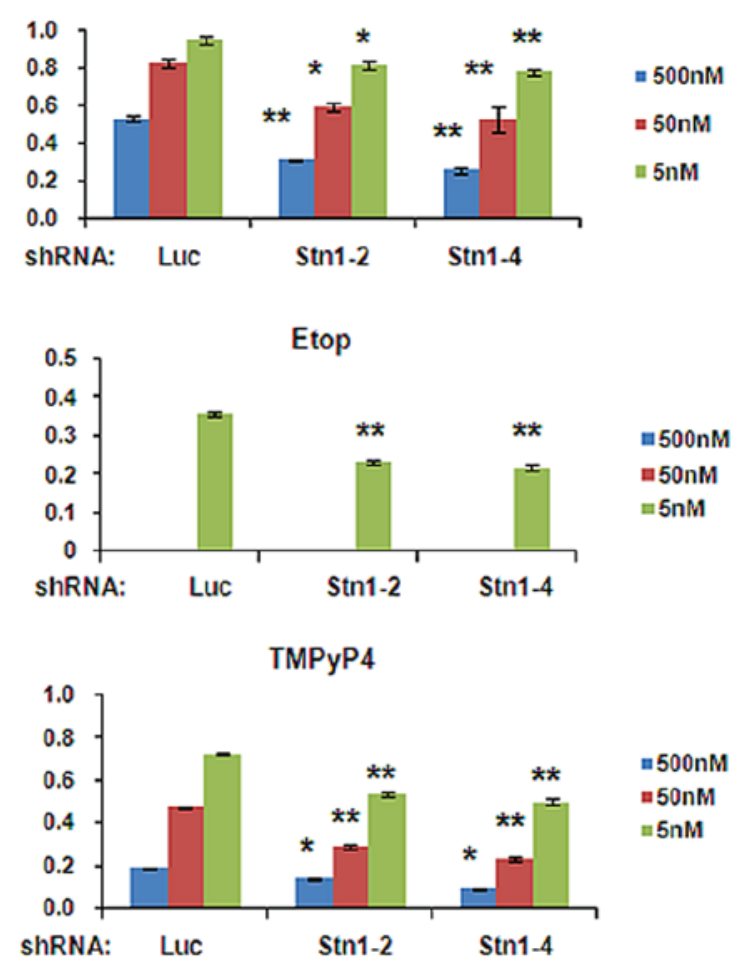

Figure 2. STN1 suppression enhances growth inhibition by various anticancer agents in the H1299 cell line. (A) Decreased colony formation of STN1 knockdown cells following exposure to CPT, Etop, Bleo and TMPyP4. The colony formation rate was calculated by dividing the number of colonies formed by drug-treated cells by the number of colonies formed by untreated cells. Each drug was tested at 5,50 and $500 \mathrm{nM}$. High concentrations of CPT or Etop (50 and $500 \mathrm{nM}$ ) caused severe cell death and no colonies were formed. The results are representative of three independent experiments. Data are presented as the mean \pm standard error. ${ }^{* *} \mathrm{P}<0.01,{ }^{*} \mathrm{P}<0.05$. (B) Reduced anchorage-independent growth ability of STN1 knockdown cells assessed by soft agar colony formation assay. Each drug was tested at 5, 50 and $500 \mathrm{nM}$. The results are representative of three independent experiments. Data are presented as the mean \pm standard error. ${ }^{* *} \mathrm{P}<0.01,{ }^{*} \mathrm{P}<0.05$. CPT, camptothecin; Etop, etoposide; Bleo, bleomycin; TMPyP4, meso-Tetra (N-methyl-4-pyridyl) porphine tetra tosylate; shRNA, small hairpin RNA; shLuc, shRNA targeting luciferase.

bonding (34). Such stabilization creates prominent barriers to DNA replication that stall replication forks, which may eventually collapse, leading to DNA damage $(35,36)$.

Following 5 days of exposure, the growth of $\mathrm{H} 1299 \mathrm{shStn1-2}$ and shStn1-4 cells was remarkably inhibited by all four agents, with $\mathrm{IC}_{50}$ values two to three times lower compared with the shLuc control cells (Table I). When the HeLa and MDA-MB231 cell lines were investigated, a similar degree of chemosensitivity was observed (Tables II and III). Therefore, STN1 knockdown reduced the survival of tumor cells following exposure to various damage-inducing anti-tumor agents, independent of cell type. 
Table II. IC $_{50}$ values of camptothecin, etoposide, bleomycin and TMPyP4 in HeLa cells with either STN1 knockdown or control.

\begin{tabular}{lcccc}
\hline & \multicolumn{3}{c}{$\mathrm{IC}_{50}$ value, $\mu \mathrm{M}$} & TMPyP4 \\
\cline { 2 - 5 } Cell type & Camptothecin & Etoposide & Bleomycin & $7.41 \pm 0.95$ \\
shLuc & $0.51 \pm 0.06$ & $0.60 \pm 0.13$ & $2.78 \pm 0.56$ & $3.54 \pm 0.72$ \\
shStn1-2 & $0.15 \pm 0.04$ & $0.24 \pm 0.09$ & $1.12 \pm 0.17$ & $3.11 \pm 1.04$ \\
shStn1-4 & $0.23 \pm 0.07$ & $0.22 \pm 0.05$ & $1.29 \pm 0.39$ & \\
\hline
\end{tabular}

$\mathrm{IC}_{50}$, half maximal inhibitory concentration; TMPyP4, meso-Tetra ( $\mathrm{N}$-methyl-4-pyridyl) porphine tetra tosylate; sh, small hairpin; shLuc, shRNA targeting luciferase.

A

혼
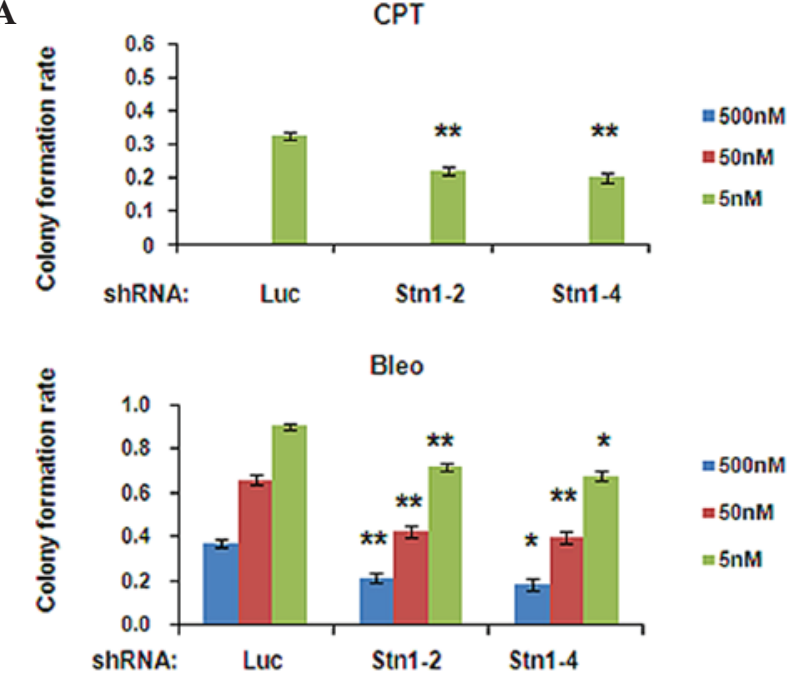

B

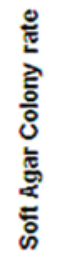
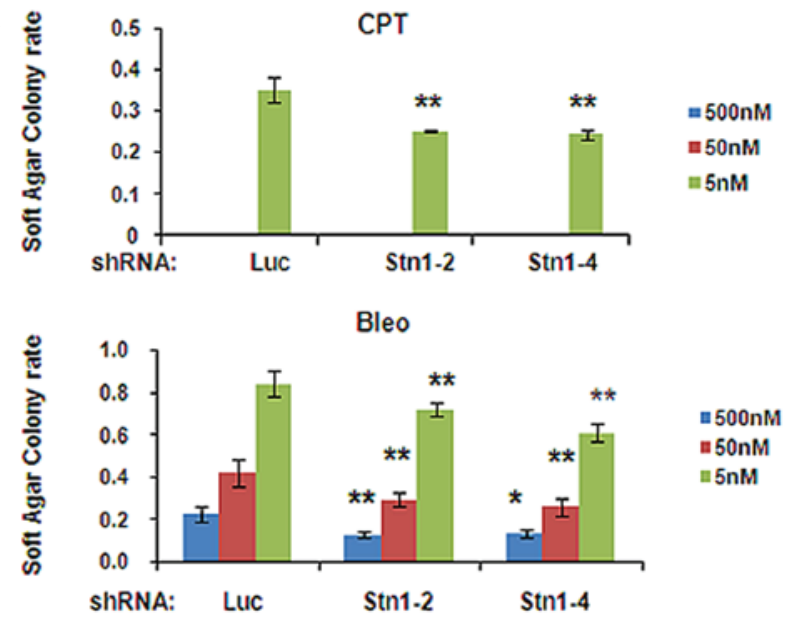

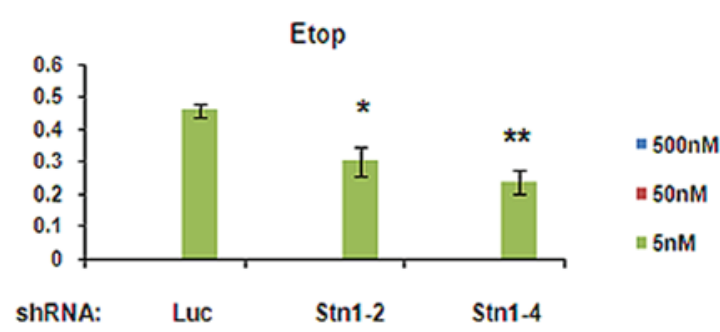

TMPyP4
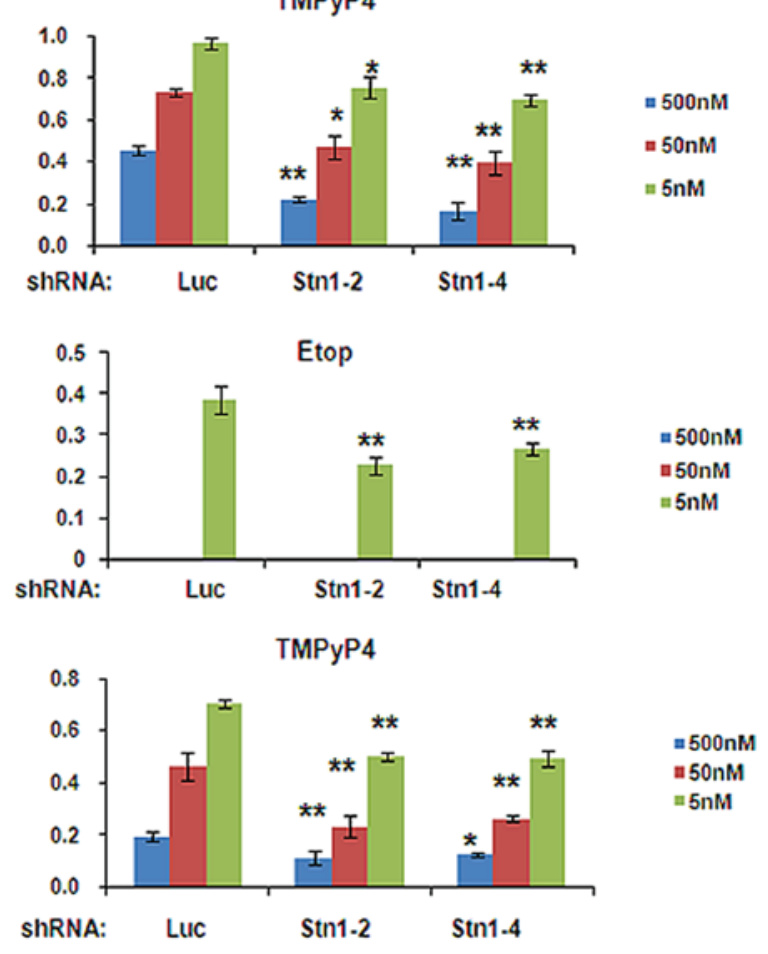

Figure 3. STN1 suppression causes increased growth-inhibitory effects by various anticancer agents in the HeLa cell line. (A) Decreased colony formation of STN1 knockdown cells following exposure to CPT, Etop, Bleo and TMPyP4. The colony formation rate was normalized to untreated cells. Each drug was tested at 5, 50 and $500 \mathrm{nM}$. The results are representative of three independent experiments. Data are presented as the mean \pm standard error. ${ }^{* *} \mathrm{P}<0.01,{ }^{*} \mathrm{P}<0.05$. (B) Reduced anchorage-independent growth ability of STN1 knockdown HeLa cells assessed by soft agar colony formation assay. Each drug was tested at 5,50 and $500 \mathrm{nM}$. The results are representative of three independent experiments. Data are presented as the mean \pm standard error. ${ }^{* *} \mathrm{P}<0.01,{ }^{*} \mathrm{P}<0.05$. CPT, camptothecin; Etop, etoposide; Bleo, bleomycin; TMPyP4, meso-Tetra (N-methyl-4-pyridyl) porphine tetra tosylate; shRNA, small hairpin RNA; shLuc, shRNA targeting luciferase.

STN1 suppression diminishes colony-formation ability and anchorage-independent growth ability in the presence of anticancer agents. To additionally investigate the effectiveness of STN1 suppression on the survival and proliferation of cancer cells, the present study evaluated whether STN1 suppression affected the abilities of cancer cells to form colonies and to grow independently of anchorage using clonogenic and soft agar assays. In both assays, the same enhanced growth inhibitory effect was observed in H1299 (Fig. 2A and B) and HeLa (Fig. 3A and B) cells with STN1 suppression following drug treatment, 
Table III. IC $_{50}$ values of camptothecin, etoposide, bleomycin and TMPyP4 in MDA-MB231 cells with either STN1 knockdown or control.

\begin{tabular}{lcccc}
\hline & \multicolumn{3}{c}{$\mathrm{IC}_{50}$ value, $\mu \mathrm{M}$} \\
\cline { 2 - 5 } Cell type & Camptothecin & Etoposide & Bleomycin & TMPyP4 \\
\hline shLuc & $0.25 \pm 0.08$ & $0.53 \pm 0.12$ & $9.33 \pm 0.76$ & $8.85 \pm 1.12$ \\
shStn1-2 & $0.12 \pm 0.13$ & $0.27 \pm 0.08$ & $7.78 \pm 1.02$ & $5.54 \pm 0.88$ \\
shStn1-4 & $0.16 \pm 0.11$ & $0.28 \pm 0.10$ & $6.59 \pm 0.85$ & $6.07 \pm 1.08$ \\
\hline
\end{tabular}

$\mathrm{IC}_{50}$, half maximal inhibitory concentration; TMPyP4, meso-Tetra (N-methyl-4-pyridyl) porphine tetra tosylate; sh, small hairpin; shLuc, shRNA targeting luciferase.

A

shLuc

shLuc

hStn1-2

$\operatorname{shStn1-4}$
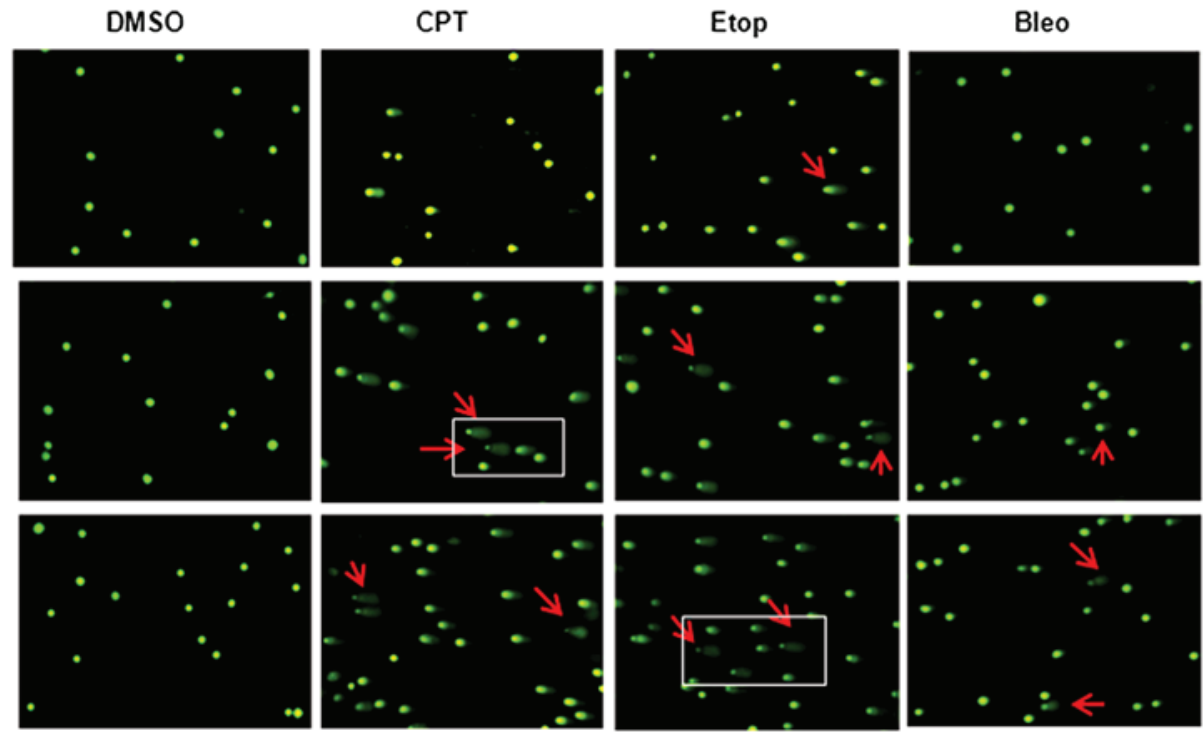
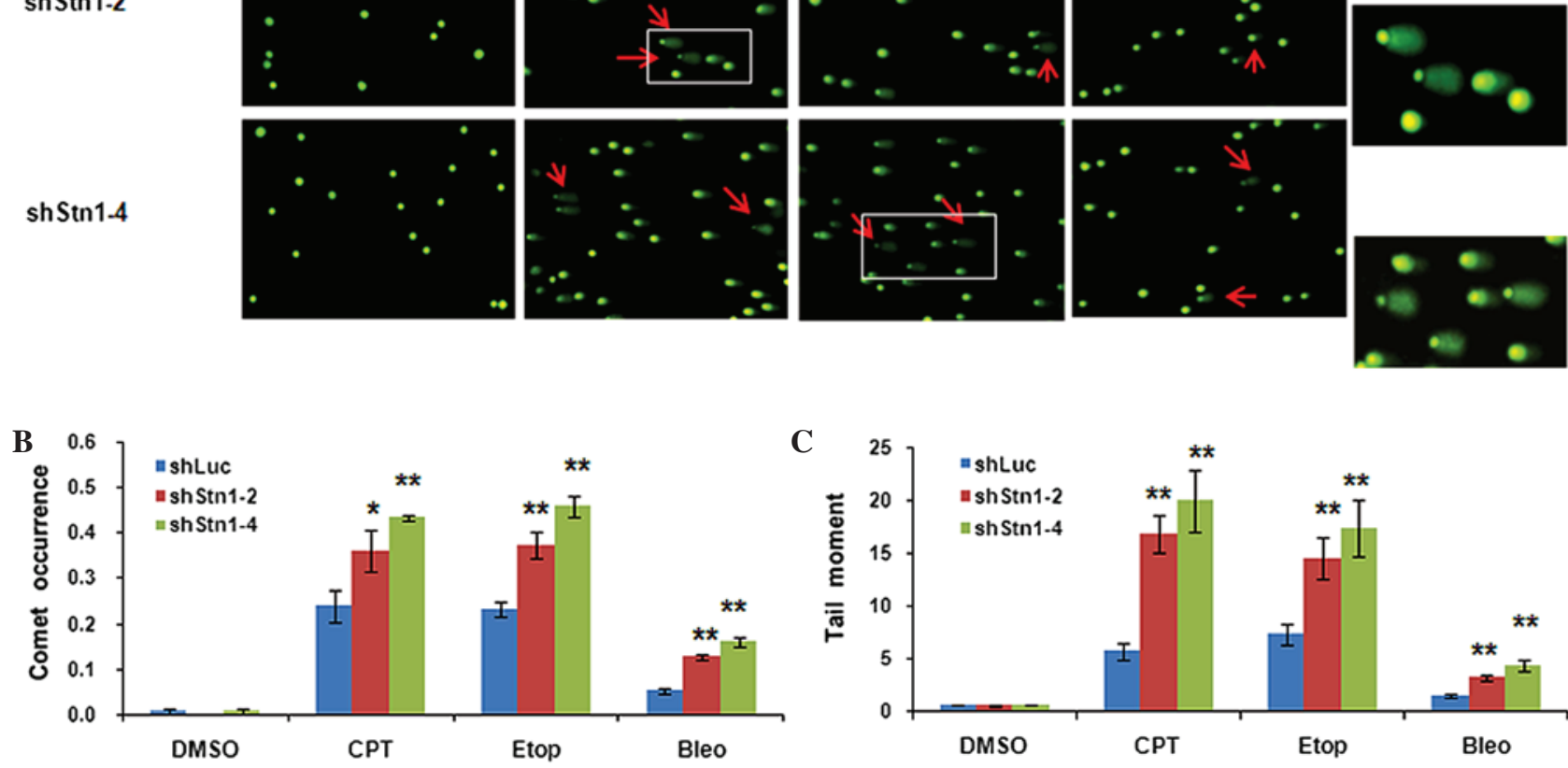

Figure 4. STN1 suppression increases DNA damage in H1299 cells. (A) Representative comet images from STN1 knockdown cells following drug treatment (CPT, Etop and Bleo). Pooled H1299 shLuc, shStn1-2 and shStn1-4 cells (2 days after puromycin selection) were treated with each drug at 1 mM for 6 h. Comet assay was performed to detect the integrity of chromosomal DNA. Arrows indicate typical DNA comets. Boxed regions are enlarged in inserts to show comets. (B) Comet occurrence and (C) tail moment are presented. Data are presented as the mean \pm standard error. ${ }^{* *} \mathrm{P}<0.01$, ${ }^{*} \mathrm{P}<0.05$. $\mathrm{CPT}, \mathrm{camptothecin}$; Etop, etoposide; Bleo, bleomycin; sh, small hairpin; shLuc, shRNA targeting luciferase; DMSO, dimethyl sulfoxide.

compared with shLuc cells. In the clonogenic assay, cells were treated with a gradient concentration of 5,50 and $500 \mathrm{nM}$ of each drug for 10 days. In the absence of drug treatment, no significant growth defects were observed in H1299 or HeLa cells with STN1 knockdown compared with the shLuc control (data not shown). Colony formation was largely inhibited by drug treatment in all cells (Figs. 2A and 3A). Notably, this inhibition was significantly escalated in STN1 knockdown H1299 cells (Fig. 2A). Under all three drug concentrations, colony numbers of drug-treated shStn1-2 and shStn1-4 H1299 cells were greatly diminished compared with the drug-treated shLuc control. The most significant changes were observed in cells treated with $500 \mathrm{nM}$ of bleomycin (shStn1-2, $\mathrm{P}=0.005$; shStn1-4, $\mathrm{P}=0.022$ ) or TMPyP4 (shStn1-2, P<0.001; shStn1-4, P<0.001; Fig. 2A). In HeLa cells, the long-term synergetic effects of STN1 knockdown and drug treatment were additionally observed. The colony growth in shStn1-2 and shStn1-4 cells was reduced in all drug-treated groups, in contrast to their shLuc controls (Fig. 3A). 
A

shLuc
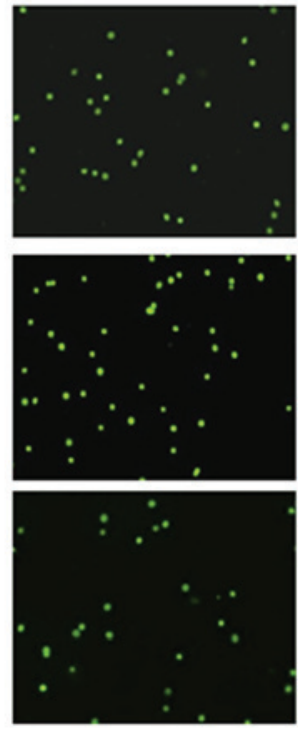

$\operatorname{shStn1-4}$
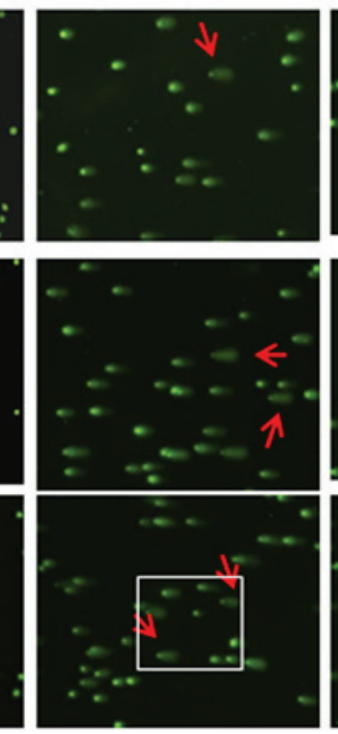

DMSO
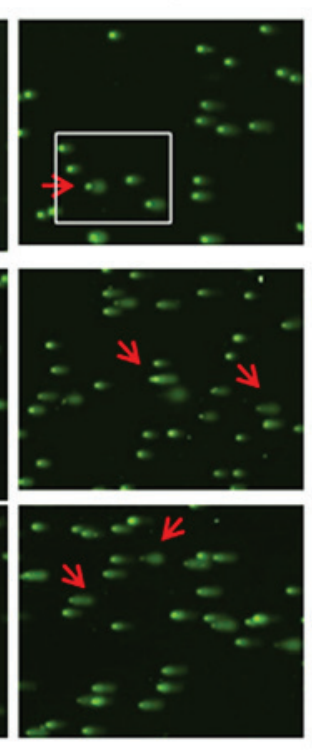

Bleo
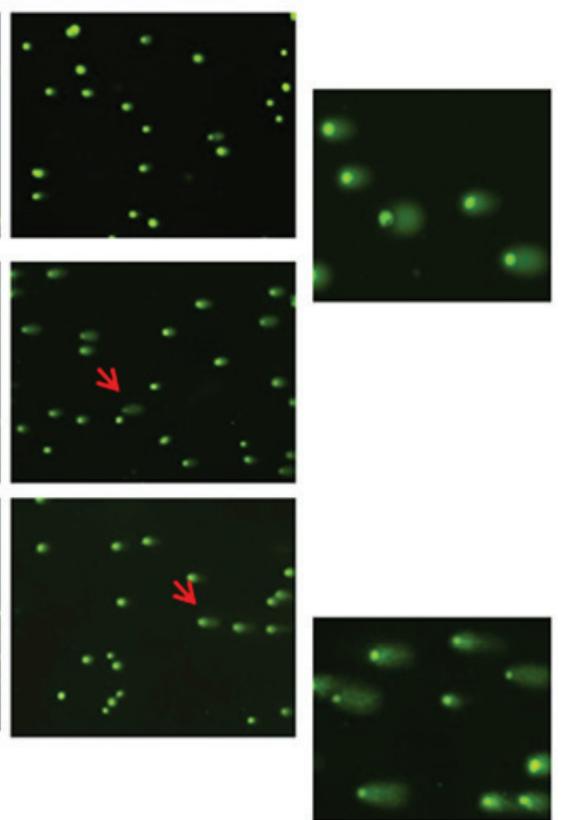
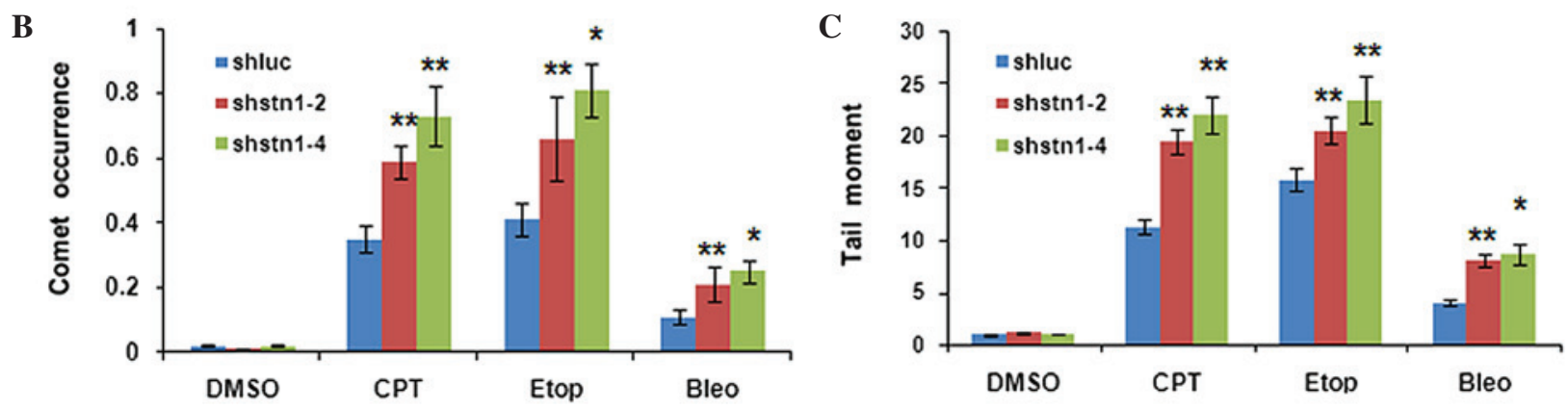

Figure 5. STN1 suppression increases DNA damage in HeLa cells. (A) Representative comet images from STN1 knockdown cells following drug treatment (CPT, Etop and Bleo). Pooled HeLa shLuc, shStn1-2 and shStn1-4 cells (2 days after puromycin selection) were treated with each drug at $3 \mathrm{mM}$ for 8 h. Comet assay was performed to detect the integrity of chromosomal DNA. Arrows indicate typical DNA comets. Boxed regions are enlarged in inserts to show comets. (B) Comet occurrence and (C) tail moment are presented. Data are presented as the mean \pm standard error. ${ }^{* *} \mathrm{P}<0.01,{ }^{*} \mathrm{P}<0.05$. CPT, camptothecin; Etop, etoposide; Bleo, bleomycin; sh, small hairpin; shLuc, shRNA targeting luciferase; DMSO, dimethyl sulfoxide.

Anchorage-independent growth is one of the hallmarks of cancerous cell transformation $(37,38)$. In general, there is a positive correlation between in vitro transformation and in vivo carcinogenesis (39-41). Soft agar assay is considered the most accurate and stringent in vitro assay for detection of malignant transformation of cells $(37,38)$. The present study used soft agar assay to determine the effect of STN1 knockdown on anchorage-independent growth in H1299 and HeLa cells. Following 10 days of treatment with drugs, STN1 knockdown enhanced growth inhibition induced by all four drugs in soft agar (Figs. $2 \mathrm{~B}$ and $3 \mathrm{~B}$ ). The results of the present study additionally support the conclusion that STN1 suppression enhanced the growth inhibitory effect of tested anticancer agents.

STN1 suppression augments DNA damage in cancer cells treated with various DNA damage inducers. Given the role of CST in maintaining genome stability and countering replication stress, it was suspected that STN1 deficiency may elevate DNA damage levels caused by damage inducers, eliciting rapid growth inhibition. To investigate this hypothesis, the present study employed the widely-used comet assay to detect DNA lesions in STN1 knockdown cells. The principle of comet assay is based on the ability of denatured broken DNA fragments to migrate out of the nucleoid under the influence of an electric field, whereas undamaged DNA migrates more slowly and remains within the confines of the nucleoid when a current is applied. Evaluation of the DNA 'comet' tail shape and migration pattern allows for assessment of DNA damage $(26,42)$. Comet assays were performed on H1299 and HeLa control cells and the corresponding STN1 knockdown cells following treatment with each chemotherapeutic agent. As shown in Fig. 4A, untreated H1299 cells in all three groups exhibited a normal organized of nucleus without tails, indicative of undamaged DNA. As expected, treating H1299 cells with damage-inducing agent (CPT, etoposide and bleomycin) induced comet tails in all groups (Fig. 4A). Notably, quantification of comet occurrence and tail moment revealed that an increased number of comets as well as longer tails were present in shStn1-2 and shStn1-4 cells compared with control shLuc cells (Fig. 4B and C). In 
CPT-treated cells, STN1 knockdown increased comet occurrence by 1.5 - $(\operatorname{shStn} 1-2, \mathrm{P}=0.011)$ and 1.8 -fold $(\operatorname{shStn} 1-4, \mathrm{P}=0.005)$. In etoposide-treated cells, shStn1-2 and shStn1-4 increased comet occurrence by 1.6- $(\mathrm{P}=0.002)$ and 2 -fold $(\mathrm{P}=0.015)$. In bleomycin-treated cells, comet occurrence in $\mathrm{H} 1299$ cells expressing shStn1-2 and shStn1-4 was 2- $(\mathrm{P}=0.001)$ and 3 -fold $(\mathrm{P}=0.001)$ higher compared with control cells (Fig. 4B). Similarly, STN1 knockdown also significantly increased the tail moment by 2 - to 3 -fold in cells treated with CPT ( $\operatorname{shStn1-2,~} \mathrm{P}=0.004$; shStn1-4, $\mathrm{P}=0.0004)$ etoposide (shStn1-2, $\mathrm{P}=0.002$; shStn1-4, $\mathrm{P}=0.003$; and bleomycin ( $\operatorname{shStn} 1-2, \mathrm{P}=0.0005 ; \operatorname{shStn} 1-4, \mathrm{P}=0.0003$; Fig. 4C).

In HeLa cells, a significant fraction of shLuc cells showed comet tails following treatment with DNA-damaging agents. However, in all drug-treated groups, shStn1-2 and shStn1-4 cells had increased numbers of comets and longer tail moments compared with shLuc controls (Fig. 5A). Among the three DNA damage inducers, etoposide gave rise to the most marked increase in terms of comet occurrence, while CPT treatment led to the largest increase in tail moments in shStn1-2 and shStn1-4 cells, in contrast to shLuc controls (Fig. 5B and C). These results suggest that STN1 knockdown cells are more susceptible to DNA damage. The results of the present study suggest that the chemosensitivity induced by STN1 suppression is at least in part due to elevated levels of DNA damage in STN1 knockdown cells.

\section{Discussion}

DNA-damaging agents have been commonly used in clinical studies to eliminate cancer cells and reduce tumor growth $(1,2,43)$. Sensitization of cancer cells to chemotherapeutic agents reduces side effects, as well as balances the efficacy and toxicity of chemotherapy (44). Given the significant role of the human CST complex in maintaining genomic stability and telomere integrity, the authors of the present study reasoned that suppression of CST expression may augment DNA damage levels, therefore sensitizing cancer cells to chemotherapeutic agents. The results of the present study revealed that suppression of STN1 increases the sensitivity of various cancer cells to several commonly used DNA damage inducing agents. Stable knockdown of STN1 using two independent shRNA sequences enhances the cytotoxicity of DNA damage inducers (camptothecin, etoposide and bleomycin) in common cancer cell lines. These results are in agreement with a previous study demonstrating that overexpression of CST proteins facilitate the recovery from replication stress-induced DNA damage (45). Mechanistically, the present study demonstrates that STN1 knockdown elevates the levels of DNA lesions induced by DNA damage inducers, implying that the enhanced cytotoxicity may be at least in part due to inefficiency in repairing DNA damage or induction of increased DNA damage by STN1 deficiency. The results of the present study provide proof of principle that combining inhibition of CST with chemotherapeutic regimens may enhance their effectiveness against malignant cell proliferation.

The results of the present study additionally suggest that the CST complex may be a novel player in the DNA repair pathway. Prior to the present study, it had only been demonstrated that the CST complex is important for countering replication stress, likely by promoting genome-wide replication restart following replication fork stalling $(9,10)$. To the best of our knowledge, no previous studies have linked CST with protecting cells from DNA damage. In fact, the results of the present comet assay reveal that there is no significant increase in DNA lesions in STN1 knockdown cells without exposure to drugs, indicating that STN1 deficiency only induces moderate DNA damage that is tolerated by cancer cells or is easily repaired. This is in agreement with previously published work demonstrating that downregulation of STN1 in cancer cells does not affect cell growth $(9,10,15)$. By contrast, following exposure to various DNA-damaging agents, DNA damage levels are markedly elevated in STN1 knockdown cells compared with control cells. The authors of the present study propose that the CST complex may have an unidentified yet significant role in repairing DNA double-strand breaks. This important function of CST remains to be elucidated in additional studies.

In conclusion, the present study demonstrates the synergistic effect of STN1 depletion and established chemotherapeutic agents. The results of the present study contribute to the knowledge concerning the function of the CST complex in cancer cell proliferation, and provide proof of principle that CST may potentially be a novel molecular target for anticancer therapy.

\section{Acknowledgements}

The present study was supported in part by the National Institute of Health (grant nos., R01GM112864 and R15GM099008 and the CONCERN Now Award to Professor Weihang Chai.

\section{References}

1. Cheung-Ong K, Giaever G and Nislow C: DNA-damaging agents in cancer chemotherapy: Serendipity and chemical biology. Chem Biol 20: 648-659, 2013.

2. Lord CJ and Ashworth A: The DNA damage response and cancer therapy. Nature 481: 287-294, 2012.

3. Bouwman P and Jonkers J: The effects of deregulated DNA damage signaling on cancer chemotherapy response and resistance. Nat Rev Cancer 12: 587-598, 2012.

4. Luo Y and Leverson JD: New opportunities in chemosensitization and radiosensitization: Modulating the DNA-damage response. Expert Rev Anticancer Ther 5: 333-342, 2005.

5. Holohan C, Van Schaeybroeck S, Longley DB and Johnston PG: Cancer drug resistance: An evolving paradigm. Nat Rev Cancer 13: 714-727, 2013.

6. Coley HM: Mechanisms and strategies to overcome chemotherapy resistance in metastatic breast cancer. Cancer Treat Rev 34: 378-390, 2008

7. Zhou BB and Bartek J: Targeting the checkpoint kinases: Chemosensitization versus chemoprotection. Nat Rev Cancer 4: 216-225, 2004.

8. Gu P, Min JN, Wang Y, Huang C, Peng T, Chai W and Chang S: CTC1 deletion results in defective telomere replication, leading to catastrophic telomere loss and stem cell exhaustion. EMBO J 31: 2309-2321, 2012.

9. Huang C, Dai X and Chai W: Human Stn1 protects telomere integrity by promoting efficient lagging-strand synthesis at telomeres and mediating C-strand fill-in. Cell Res 22: 1681-1695, 2012.

10. Stewart JA, Wang F, Chaiken MF, Kasbek C, Chastain PD II, Wright WE and Price CM: Human CST promotes telomere duplex replication and general replication restart after fork stalling. EMBO J 31: 3537-3549, 2012.

11. Casteel DE, Zhuang S, Zeng Y, Perrino FW, Boss GR, Goulian M and Pilz RB: A DNA polymerase-\{alpha\} \{middle dot\}primase cofactor with homology to replication protein A-32 regulates DNA replication in mammalian cells. J Biol Chem 284: 5807-5818, 2009. 
12. Wan M, Qin J, Songyang Z and Liu D: OB-fold containing protein 1 (OBFC1), a human homolog of yeast Stn1, associates with TPP1 and is implicated in telomere length regulation. J Biol Chem 284: 26725-26731, 2009.

13. Chen LY, Majerská J and Lingner J: Molecular basis of telomere syndrome caused by CTC1 mutations. Genes Dev 27: 2099-2108, 2013.

14. Anderson BH, Kasher PR, Mayer J, Szynkiewicz M, Jenkinson EM, Bhaskar SS, Urquhart JE, Daly SB, Dickerson JE, O'Sullivan J, et al: Mutations in CTC1, encoding conserved telomere maintenance component 1 , cause Coats plus. Nat Genet 44: 338-342, 2012.

15. Chen LY, Redon S and Lingner J: The human CST complex is a terminator of telomerase activity. Nature 488: 540-544, 2012.

16. Wang F, Stewart JA, Kasbek C, Zhao Y, Wright WE and Price CM Human CST has independent functions during telomere duplex replication and C-strand fill-in. Cell Rep 2: 1096-1103, 2012.

17. Wu P, Takai $\mathrm{H}$ and de Lange T: Telomeric 3 ' overhangs derive from resection by Exol and Apollo and fill-in by POT1b-associated CST. Cell 150: 39-52, 2012.

18. Shay JW and Wright WE: Telomerase therapeutics for cancer: Challenges and new directions. Nat Rev Drug Discov 5: 577-584, 2006.

19. De Cian A, Lacroix L, Douarre C, Temime-Smaali N, Trentesaux C, Riou JF and Mergny JL: Targeting telomeres and telomerase. Biochimie 90: 131-155, 2008.

20. Mondello C and Scovassi IA: Telomeres, telomerase, and apoptosis. Biochem Cell Biol 82: 498-507, 2004.

21. Chan SR and Blackburn EH: Telomeres and telomerase. Philos Trans R Soc Lond B Biol Sci 359: 109-121, 2004.

22. Liu Y, Bohr VA and Lansdorp P: Telomere, telomerase and aging. Mech Ageing Dev 129: 1-2, 2008

23. Chen $M$ and McLeskey SW: Telomere-based cancer treatment: emerging targeted therapies. Clin J Oncol Nursing 14: 720-727, 2010 .

24. Herbert BS, Gellert GC, Hochreiter A, Pongracz K, Wright WE, Zielinska D, Chin AC, Harley CB, Shay JW and Gryaznov SM: Lipid modification of GRN163, an N3'-P5' thio-phosphoramidate oligonucleotide, enhances the potency of telomerase inhibition. Oncogene 24: 5262-5268, 2005.

25. Dai X, Huang C, Bhusari A, Sampathi S, Schubert K and Chai W: Molecular steps of G-overhang generation at human telomeres and its function in chromosome end protection. EMBO J 29: $2788-2801,2010$

26. Collins AR: The comet assay. Principles, applications, and limitations. Methods Mol Biol 203: 163-177, 2002.

27. Gu P and Chang S: Functional characterization of human CTC1 mutations reveals novel mechanisms responsible for the pathogenesis of the telomere disease Coats plus. Aging Cell 12: 1100-1109, 2013.

28. Pizarro JG, Folch J, Junyent F, Verdaguer E, Auladell C, Beas-Zarate C, Pallàs $\mathrm{M}$ and Camins $\mathrm{A}$ : Antiapoptotic effects of roscovitine on camptothecin-induced DNA damage in neuroblastoma cells. Apoptosis 16: 536-550, 2011.

29. Berniak K, Rybak P, Bernas T, Zarebski M, Biela E, Zhao H, Darzynkiewicz Z and Dobrucki JW: Relationship between DNA damage response, initiated by camptothecin or oxidative stress, and DNA replication, analyzed by quantitative 3D image analysis. Cytometry A 83: 913-924, 2013.
30. Nguyen TV, Chen JK and Murray V: Bleomycin DNA damage: Anomalous mobility of 3'-phosphoglycolate termini in an automated capillary DNA sequencer. J Chromatogr B Analyt Technol Biomed Life Sci 913-914: 113-122, 2013.

31. Patel JR, Dhorajiya BD, Dholakiya BZ, Badria FA and Ibrahim AS: In-vitro cytotoxicity, antioxidant, bleomycin-dependent DNA damage and immunomodulatory evaluation of 1-(4-acetylphenyl)-3-aryloxypyrrolidine-2, 5-dione based derivatives. Med Chem Res 23: 3907-3915, 2014.

32. Li X, Liu W, Wang H, Yang L, Li Y, Wen H, Ning H, Wang J, Zhang L, Li J and Fan D: Rap1 is indispensable for TRF2 function in etoposide-induced DNA damage response in gastric cancer cell line. Oncogenesis 4: e144, 2015.

33. Griaud F, Williamson AJK, Taylor S, Potier DN, Spooncer E, Pierce A and Whetton AD: BCR/ABL modulates protein phosphorylation associated with the etoposide-induced DNA damage response. J Proteomics 77: 14-26, 2012.

34. Grand CL, Han H, Muñoz RM, Weitman S, Von Hoff DD, Hurley LH and Bearss DJ: The cationic porphyrin TMPyP4 down-regulates c-MYC and human telomerase reverse transcriptase expression and inhibits tumor growth in vivo. Mol Cancer Ther 1: 565-573, 2002.

35. Tarsounas $\mathbf{M}$ and Tijsterman $\mathrm{M}$ : Genomes and G-quadruplexes: For better or for worse. J Mol Biol 425: 4782-4789, 2013.

36. Bochman ML, Paeschke K and Zakian VA: DNA secondary structures: Stability and function of G-quadruplex structures. Nat Rev Genet 13: 770-780, 2012.

37. Borowicz S, Scoyk MV, Avasarala S, Karuppusamy Rathinam MK, Tauler J, Bikkavilli RK and Winn RA: The soft agar colony formation assay. J Vis Exp 92: e51998, 2014.

38. Horibata S, Vo TV, Subramanian V, Thompson PR, Coonrod SA: Utilization of the soft agar colony formation assay to identify inhibitors of tumorigenicity in breast cancer cells. J Vis Exp 99: e52727, 2015.

39. IARC/NCI/EPA Working Group: Cellular and molecular mechanisms of cell transformation and standardization of transformation assays of established cell lines for the prediction of carcinogenic chemicals: Overview and recommended protocols. Cancer Res 45: 2395-2399, 1985.

40. Kolber AR, Wong TK, Grant LD, DeWoskin RS and Hughes TJ (eds): In Vitro Toxicity Testing of Environmental Agents. Current and Future Possibilities Part A: Survey of Test Systems. Plenum Press, New York, pp321-322, 1979.

41. Pullman B, Ts'O POP and Schneider EL (eds): Interrelationship Among Aging, Cancer and Differentiation. The Jerusalem Symposia on Quantum Chemistry and Biochemistry. Volume 18. D. Reidel Publishing Company, Dordrecht, The Netherlands, pp247-248, 1985

42. Collins AR: The comet assay for DNA damage and repair: Principles, applications, and limitations. Mol Biotechnol 26: 249-261, 2004.

43. Hosoya $\mathrm{N}$ and Miyagawa K: Targeting DNA damage response in cancer therapy. Cancer Sci 105: 370-388, 2014.

44. Dickerson EB, Blackburn WH, Smith MH, Kapa LB, Lyon LA and McDonald JF: Chemosensitization of cancer cells by siRNA using targeted nanogel delivery. BMC Cancer 10: 10, 2010.

45. Wang F, Stewart J and Price CM: Human CST abundance determines recovery from diverse forms of DNA damage and replication stress. Cell Cycle 13: 3488-3498, 2014. 